\title{
Tratamiento de la documentación audio- visual en el entorno digital: iniciativas de metadatos y lenguajes de descripción multimedia
}

\author{
Por Alfonso López Yepes; Rodrigo Sánchez Jiménez \\ y José Ramón Pérez Agüera
}

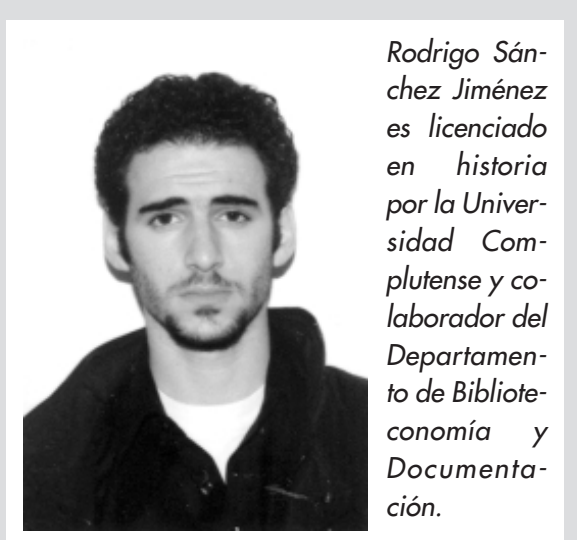

Resumen: Se ofrece al lector una perspectiva general de las principales iniciativas que se están llevando a cabo en materia de documentación audiovisual en formato digital. Para ello se hace un repaso por los estándares diseñados por las instituciones normalizadoras y empresas de multidifusión, entre los que destacan Mpeg-7, Smil, RDF, Dublin core, TV-Anytime. Así mismo se describe brevemente el Diccionario de Metadatos de Smpte con un énfasis especial en algunos de sus elementos más importantes como KLV y Umid. También se hace un repaso a las iniciativas llevadas a cabo en el ámbito de las bibliotecas y archivos describiendo brevemente ViDE, Mets, Data dictionary for audio/video metadata y el proyecto del Nedlib. Para finalizar se comenta un ejemplo de aplicación de los estándares descritos dentro del ám-

Alfonso López Yepes es catedrático de documentación, director del Departamento de Biblioteconomía y Documentación de la Universidad Complutense y director de la revista Cuadernos de documentación multimedia. Dirige el proyecto "Red iberoamericana de documentación informativa (InfoDocNet)" financiado por el Vicerrectorado de Estudios de la UCM.

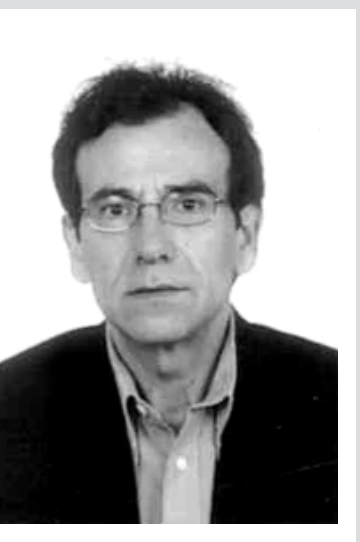
bito de la Red Iberoamericana de Documentación Informativa, donde se está intentando componer un marco empírico de adaptación de los mismos.

Palabras clave: Documentación audiovisual, Mpeg-7, RDF, Dublin Core, Smil, $T V$-Anytime, Smpte, Smef, EBU, Mets.

Title: The handling of audio-visuals in the digital environment: initiatives on metadata and languages for multimedia descriptions

Abstract: This article offers the reader a broad perspective of the main initiatives relating to digital audio-visual documentation. To this end a review is made of the standards designed within both the broadcasting industry and standards organisations. Among the standards highlighted are Mpeg-7, Smil, rdf, Dublin core and TV-Anytime. The Smpte metadata dictionary is also described, with special emphasis on those elements of greater importance, such as KLV and Umid. Subsequently, certain initiatives carried out within the area of libraries and archives are mentioned with short descriptions provided of ViDE, Mets, Data Dictionary for Audio/Video Metadata and the Nedlib project. The article concludes with an example of how the standards described have been applied within the environment of the "Red Iberoamericana de Documentacion Multimedia" - Iber-American Network of Multimedia Documentation-, where a framework is being developed for an empirical testing of the above mentioned standards.

Keywords: Audio-visual documentation, Mpeg-7, Rdf, Dublin core, Smil, TV-Anytime, Smpte, Smef, EBU, Mets.

López Yepes, Alfonso; Sánchez Jiménez, Rodrigo; Pérez Agüera, José Ramón. "Tratamiento de la documentación audiovisual en el entorno digital: iniciativas de metadatos y lenguajes de descripción multimedia". En: El profesional de la información, 2003, noviembre-diciembre, v. 11, n. 6, pp. 443-451.

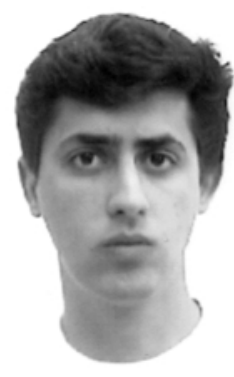

José Ramón Pérez Agüera es licenciado en historia por la Universidad Complutense, colaborador del Departamento de Biblioteconomía y Documentación y becario de formación de personal investigador de la Comunidad de Madrid. 


\section{Introducción}

El continuo avance de las nuevas tecnologías en los últimos años ha afectado de forma muy especial al ámbito de la documentación audiovisual. Los procesos de digitalización y la capacidad de internet como nuevo medio de multidifusión, han provocado que las empresas del sector hayan tomado conciencia de la revolución que se planteaba y hayan querido tomar la iniciativa en proyectos que faciliten tanto el proceso de producción como el de difusión audiovisual y permitan su adaptación al nuevo entorno tecnológico que está surgiendo, incrementando su capacidad de intercambio y gestión.

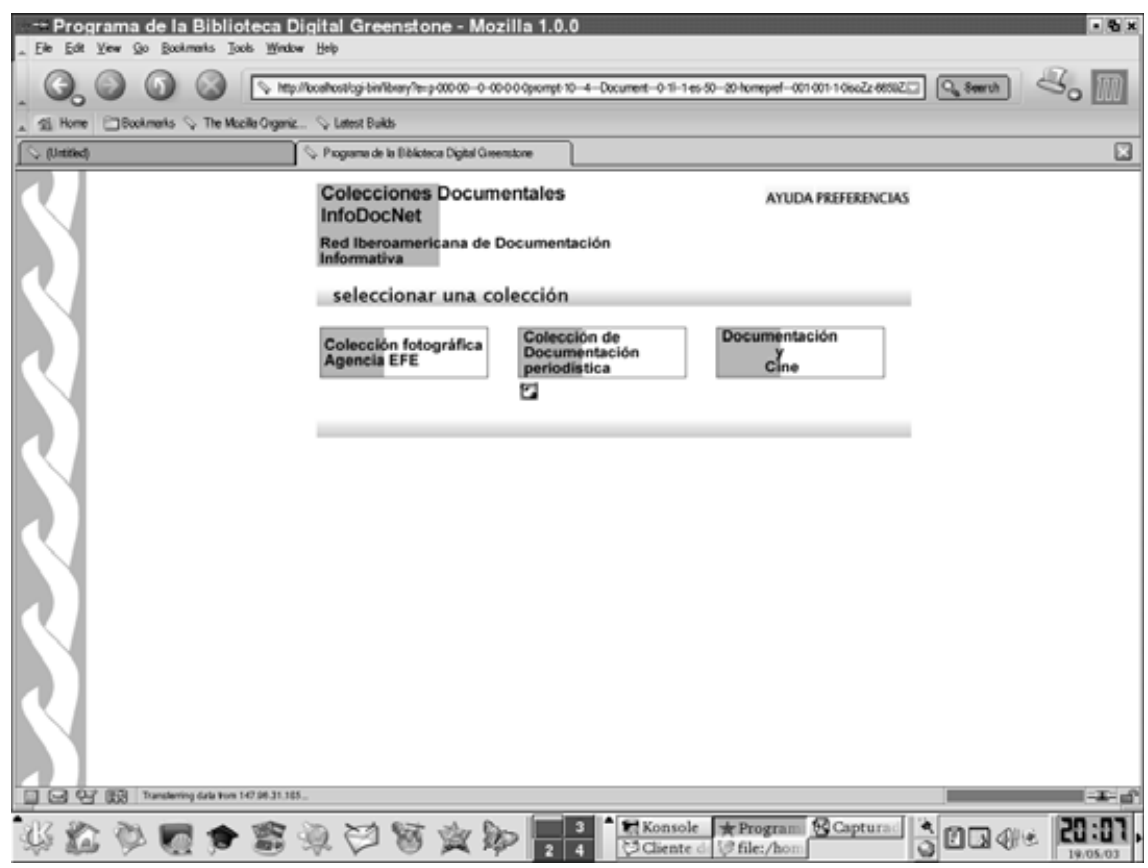

A su vez, los archivos de lo audiovisual y las bibliotecas, muchos de ellos con serios problemas de conservación, han visto en la digitalización una necesidad por el número creciente de documentos en formatos digitales que están llegando a sus fondos, y una posible salida de cara a la conservación y preservación de materiales que de otra forma están condenados a la desaparición.

"Se vienen desarrollando varios proyectos por parte de las grandes asociaciones de empresas de multidifusión, centradas en la normalización y estandarización de metadatos que permitan de forma efectiva el intercambio de materiales»

$\mathrm{Al}$ contrario que las televisiones, donde los criterios fundamentales de digitalización giran en torno a la mejora de la difusión o la comercialización de sus fondos audiovisuales, el caso de los archivos audiovisuales está unido a una concepción en la que la rentabilidad cultural es el principal impulso. Este es el caso del Ndiipp (National Digital Information Infrastructure and Preservation Program) de la Library of Congress ${ }^{1}$, o de otros proyectos piloto más cercanos, como el de Patrimonio.es uno de cuyos objetivos iniciales es el de la digitalización de los fondos de Filmoteca Española .

En las actividades de análisis de contenidos audiovisuales se debería distinguir entre metadatos, los cuales nos permiten elaborar desde una perspectiva semántica una serie de datos sobre el documento que pretendemos describir, y leguajes de representación, que nos dotan de una sintaxis con la cual expresar los contenidos semánticos representados por los metadatos. En las siguientes líneas pretendemos ofrecer una visión general de la utilización de ambos, así como de las combinaciones sintaxis/semántica más habituales.

No trataremos aquí más que de forma muy tangencial el problema de los formatos de compresión y edición de vídeo, debido a que su amplitud requeriría un artículo de las mismas dimensiones que éste.

\section{Metadatos y documentación audiovisual}

Cuando hablamos de metadatos en el entorno audiovisual debemos tener en cuenta que no podemos trabajar de la misma forma que cuando los utilizamos referidos a documentos textuales. Esto es debido a que la complejidad de los procesos de producción, edición, difusión y archivo dentro del campo de la documentación audiovisual es muy superior y cuenta con muchas más variables que en el caso de la escrita. La cantidad de datos que se generan durante todo el proceso de tratamiento de la documentación audiovisual nos obliga a tener en cuenta una gran cantidad de información añadida que acompaña a los contenidos del documento audiovisual y cuyo interés de cara a la recuperación puede ser tan alto, en determinadas circunstancias, como el que tienen los propios contenidos del documento. Metadatos que tienen que ver con aspectos técnicos, por ejemplo, son fundamentales en entornos profesionales para el tratamiento, difusión, transferencia y almacenamiento de los materiales y por ello deben ser contemplados por el documentalista de cara a una correcta gestión de los materiales audiovisuales que se encuentran en un archivo. Lo mismo ocurre con aquellos metadatos relacionados con cuestiones legales, 
fundamentales en ámbitos como la televisión para una utilización efectiva de los documentos que residen en el archivo.

De forma muy general, Kashyap y otros (1997) proponen una clasificación de los metadatos en el entorno audiovisual basada en el tipo de relación que existe entre los mismos y los contenidos del documento. Se distingue entre los independientes del contenido, es decir, que no están relacionados con el contenido sino con el documento en sí, (fecha de creación, formato, etc.) y dependientes del contenido. A su vez, entre estos últimos puede diferenciarse entre los basados en el contenido directo (índices de texto completo) y aquellos que son descriptivos del contenido, dentro de los cuales se encuentran, entre otros, los que son producto del trabajo de indización y resumen llevado a cabo por el documentalista. Para finalizar, los autores diferencian dos subcategorías más entre los metadatos que son descriptivos del contenido del documento: independientes del dominio y específicos del mismo. Los primeros se refieren a los que no dependen del área de aplicación o temática del documento, como por ejemplo definiciones de documentos sgml; mientras que los segundos sí están relacionados con la temática, y son los que normalmente suelen contar con un tesauro específico, ontologías o diccionarios de autori$\mathrm{dad}^{4}$.

\section{Propuestas concretas de metadatos en el en- torno audiovisual.}

En los esfuerzos de estandarización de un modelo de metadatos para el campo audiovisual hemos de diferenciar entre los grupos de trabajo que han surgido en el contexto de las empresas audiovisuales (televisiones, productoras, etc.) centrados en producción, edición, difusión, almacenamiento e intercambio de la documentación audiovisual; y aquellos que han aparecido en torno a los archivos y bibliotecas cuyo principal interés es la descripción del contenido, catalogación, conservación y preservación de los materiales audiovisuales.

a. Iniciativas de empresas.

La consideración del material de archivo como una parte fundamental del capital de las empresas de lo au-

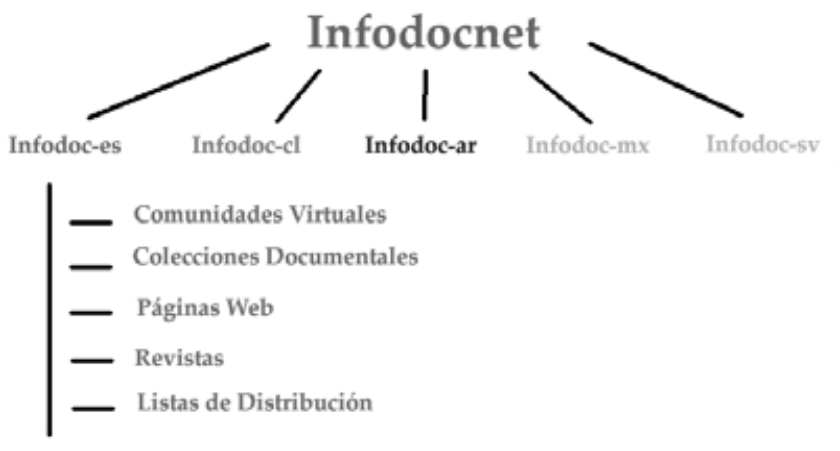

diovisual, unida a la necesidad creciente de establecer una forma de trabajo distribuido dentro de las mismas organizaciones, ha planteado la necesidad de establecer estándares normalizados comunes que permitan el intercambio de materiales audiovisuales tanto en el interior de una institución como hacia el exterior, ya sea a nivel nacional o internacional.

Por esta razón desde hace ya algunos años se vienen desarrollando varios proyectos por parte de las grandes asociaciones de empresas de multidifusión, centradas en la normalización y estandarización de metadatos que permitan de forma efectiva el intercambio de materiales. A continuación trataremos algunos de los más importantes y que nos han servido de apoyo para el diseño del modelo que estamos aplicando en la Red Iberoamericana de Documentación Informativa (InfoDocNet).

-PMC Project P/Meta (Metadata exchange standards $)^{5}$.

Ha sido creado dentro de la EBU (European Broadcasting Union). Se trata de un grupo de trabajo que tiene como principal objetivo la normalización de una serie de estándares destinados a facilitar el intercambio de materiales entre las distintas empresas que componen $E B U$. Para la realización de este propósito, $P / M e$ $t a$ se basa en el desarrollo de Smef (Standard media exchange framework) diseñado en principio por la $B B C$ como una estructura de metadatos y un modelo de datos enfocados al proceso de producción. La intención de P/Meta es fusionar Smef con el diccionario de metadatos EBU-Smpte, del que hablaremos más adelante, con la intención de ampliar su potencialidad y ofrecer a las empresas del ramo un entorno de descripción unificado.

Asimismo, $P /$ Meta está trabajando en el establecimiento de identificadores únicos como Umid (Unique material identifier), del que también hablaremos en los próximos párrafos como método de intercambio y reconocimiento eficaz.

\section{- Smpte (Society of Moving Pictures and Televi- sion Engineers).}

Mientras que en el caso anterior se investigaba en el desarrollo de un sistema de permita el intercambio de materiales entre las distintas empresas relacionadas en el contexto de la multidifusión, el grupo de trabajo sostenido por Smpte tiene como principal objetivo la elaboración de un estándar de metadatos alrededor de los procesos de producción de material audiovisual en entornos televisivos. De este trabajo ha surgido el Diccionario de metadatos Smpte, cuyo diseño se ha cuidado para que sirva a las distintas empresas, independientemente de sus propios sistemas de producción, 


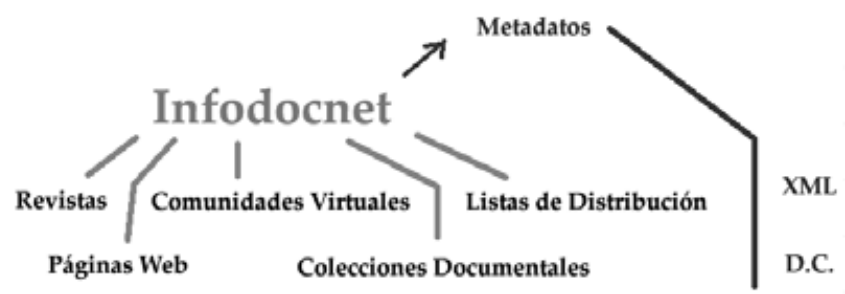

así como para que se adapte con comodidad al estándar ISO Mpeg-7.

El diccionario trata de ser una referencia de descriptores audiovisuales que cubra todo el proceso de producción. De esta forma abarca preproducción, postproducción, compra, distribución, trasmisión, almacenamiento y archivo. Los criterios que se han seguido para su elaboración han sido la definición de un esquema general que permita el registro jerárquico de los elementos que constituyen los metadatos, basada en una etiqueta universal y la harmonización de los diversos conjuntos de metadatos, teniendo en cuenta criterios de compatibilidad con el resto de iniciativas al respecto. Está compuesto por 10 clases distintas, entre las que encontramos de tipo administrativo, identificativo, técnico, relacional, espacio-temporal, de procesamiento, interpretativo, etc. Existe también una clase experimental destinada a acoger metadatos que aún no han sido incluidos en ninguna de las clases predefinidas.

Paralelamente otros proyectos han elaborado escenarios unificados de intercambio para superar los problemas de incompatibilidad técnica y de identificación de materiales de manera unívoca, que han dado como resultado los estándares $K L V$ (Key length value), $A A F$ (Advanced authoring format) y Umid.

$K L V$ es un protocolo que define una estructura de datos independiente de la aplicación o del método de trasporte usado y que por lo tanto permite el intercambio de documentos audiovisuales y de sus metadatos de forma fluida entre diferentes entornos. Su integración con Smpte es total, hasta el punto de que el diccionario contempla un grupo de metadatos específicos para la descripción de este protocolo.

Por otro lado, el $A A F$ trata de solventar otro de los problemas fundamentales que surgen a la hora de intercambiar documentos entre instituciones, que no es otro que el problema de los formatos. Es una aplicación de código abierto destinada a facilitar el trabajo con grandes volúmenes de documentos y con sus metadatos asociados. Está específicamente desarrollada para el proceso de producción digital y puede manejar diferentes tipos de documentos en distintos formatos. Su gran ventaja es que, gracias a su diseño modular, permite una continua escalabilidad y por lo tanto el tratamiento de nuevos formatos. Puede ser descargada gratuitamente de internet desde la web de Source Forge ${ }^{6}$.
Por último, otra de las iniciativas destacables en este complejo proceso de normalización es Umid, la cual también está estrechamente relacionada con los proyectos de normalización de metadatos para el entorno digital llevados a cabo por la EBU y el Smtpe. Umid forma parte de la primera clase del diccionario de metadatos Smtpe, aquella relacionada con la identificación y localización de los documentos audiovisuales.

Umid surge de la ya antigua necesidad de identificar de forma unívoca un documento independientemente del ámbito en el que nos encontremos. En el caso de la documentación audiovisual en televisión, puede referirse tanto a conjuntos de contenidos ya elaborados como a materiales "brutos". Además, permite no sólo la identificación de los documentos a través de un código específico, sino también la relación entre los documentos y sus metadatos a través del citado código.

\section{«Mets proporciona un mecanis- mo útil para la utilización de metadatos de tipo administrati- vo, descriptivo y estructural»}

Por último es interesante mencionar TV-Anytime, una iniciativa procedente de la agrupación de varias empresas de multidifusión, fundamentalmente en el negocio de la televisión digital a la carta. El proyecto, que comenzó en septiembre de 1999 y que hoy en día se encuentra ya en su segunda fase, se ha centrado en generar un modelo de metadatos de utilidad para la descripción de programas de televisión de cara al desarrollo de guías de programación tanto para usuarios como para el conjunto de actores del sector interesados en el intercambio o la compra de contenidos. Durante la primera fase se ha desarrollado un modelo para la recuperación de programación, su captura y reproducción, a la vez que la segmentación e indización de los contenidos. A lo largo de la segunda etapa se tratará de construir estándares abiertos sobre la base desarrollada durante la primera fase incluyendo aspectos como la redistribución y el tratamiento de nuevos tipos de contenidos.

Desde sus inicios, este proyecto ha mantenido una colaboración muy estrecha con el resto de iniciativas de documentación digital, como Mpeg-7 y el trabajo de grupo P/Meta de la $E B U$, lo cual garantiza una completa adaptabilidad con el resto de los que se están desarrollando y lo convierte en un complemento de gran utilidad para este sector tan especializado.

b. Iniciativas de metadatos de archivos y bibliotecas.

Una vez se han visto de forma sucinta cuáles son las vías principales que se están siguiendo en la elaboración de estándares para metadatos en producción au- 
diovisual es interesante ver también, aunque sea brevemente, qué iniciativas se están llevando a cabo en relación con la documentación audiovisual en el ámbito de las bibliotecas y archivos, donde destacan varias de ellas de gran interés.

La primera es Video development initiative (ViDE) y su User's guide: Dublin core application profile for digital video. Este proyecto trata de realizar un perfil de aplicación para el vídeo digital, el cual comprende metadatos de tipo administrativo y estructural. Para la realización de este modelo se han basado en la norma general propuesta por Dublin core con la intención de ofrecer un esquema con el que describir la creación, difusión, el acceso y el almacenamiento de documentos audiovisuales en formato digital.

Dentro de un espectro más relacionado con las bibliotecas tenemos Data dictionary for audio/video metadata, elaborado en el entorno de la Library of Congress de EUA, dentro de un proyecto de preservación de documentos audiovisuales en formato digital, siendo su principal objetivo diseñar un sistema de referencia sobre su contenido. Comprende campos que facilitan el almacenamiento y la gestión de las colecciones de tipo audiovisual.

En relación más estrecha con la preocupación por la preservación - la conservación a largo plazo de los archivos audiovisuales en formato digital- el Nedlib (Networked European deposit library) ha realizar un informe que define los metadatos mínimos requeridos. El conjunto establecido tiene una especial importancia de cara a su utilización en museos y filmotecas, los cuales son responsables de una cantidad creciente de documentos en formato digital en la perspectiva tecnológica en continua evolución. El esquema de meta- datos propuesto se compone de 8 elementos y 38 subelementos encargados de describir, entre otras características, los requerimientos técnicos necesarios para almacenar y reproducir los documentos digitales.

Por último, otro proyecto de relevancia en el ámbito de las bibliotecas y archivos relacionado con la conservación de documentos digitales que contempla el tratamiento de documentación audiovisual es Mets (Metadata encoding and transmission standard) elaborado por la Digital Library Federation. En este caso se trata de un lenguaje basado en xml destinado a proporcionar la sintaxis necesaria para la gestión de metadatos en una biblioteca digital, facilitando la gestión e intercambio de los documentos. El esquema que propone Mets es un mecanismo útil para la utilización de metadatos de tipo administrativo, descriptivo y estructural, con lo que se está consolidando como un sistema apropiado para el intercambio de documentos digitales entre bibliotecas tanto de tipo audiovisual como textual.

\section{Modelos de descripción de documentos audiovisuales}

\section{Mpeg-7.}

Uno de los que tiene más empuje es el propuesto por el veterano Moving picture experts group, también conocido como Multimedia content description interface. Es un estándar ISO/IEC para la descripción de recursos audiovisuales, útil tanto para la utilización directa por parte de humanos como para el procesamiento automático o semiautomático. Crece sobre los anteriores estándares del grupo $\mathrm{Mpeg}$, los de codificación Mpeg-1 y Mpeg-2 para el vídeo interactivo en cd-rom, el DVD y la televisión digital; y Mpeg-4, el estándar de codificación para documentos multimedia basado en objetos. Sin embargo, y a pesar

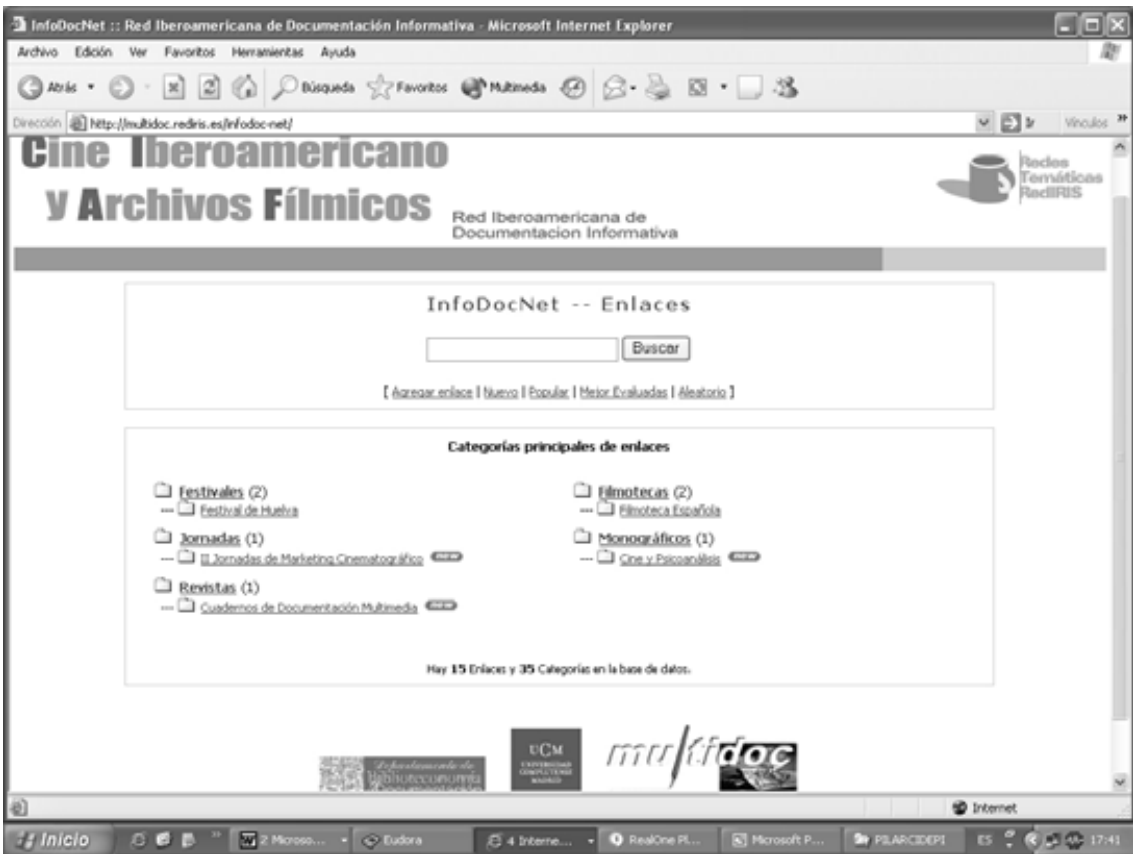
de que se puede embeber en ellos a través de wrappers (empaquetados o envoltorios), de lo que hablaremos más tarde, Mpeg-7 no es en sí un estándar de codificación sino de descripción de contenidos, un medio para representar documentos audiovisuales en sus diversas facetas.

$\mathrm{Su}$ alcance es amplio, ya que da cabida a imágenes estáticas, 3D, audio, vídeo y combinaciones de varios formatos. Además, ya que ha sido diseñado para ser un estándar, intenta adaptarse al mayor número de aplicaciones y ámbitos de utilización. Para proporcionar un marco de descripción flexible establece un conjunto de descriptores para repre- 
sentar las diferentes características de los documentos y una serie de esquemas que proporcionan la estructura, semántica y relaciones entre descriptores y entre descriptores y esquemas. Esto es posible gracias a un DDL (Description definition language) que es fundamentalmente una versión extendida y modificada de $X m l$ Schema que facilita un entorno de trabajo adaptado a las especiales necesidades (multimedia) de $\mathrm{Mpeg}$ 7.

Una de las características peculiares de Mpeg-7 es que ha sido diseñado para que la información relacionada con un documento pueda estar o no integrada en éste. Esto es posible gracias a que se puede codificar Mpeg-7 en formato binario (inteligible por máquinas aunque no por humanos), lo que mediante el uso de los citados wrappers permite que se conserven en un mismo archivo tanto el documento como los metadatos referentes al mismo. Este tipo de codificación es una mezcla entre compresión y análisis (parsing o parseado) de los documentos xml, lo que facilita mucho el procesamiento automatizado de documentos. También es posible mantener los datos descriptivos disociados del documento al que hacen referencia, ya sea en formato binario como textual (y por tanto legible por humanos), lo que permite tratar solamente los documentos descriptivos sin necesidad de tener que procesar documentación audiovisual propiamente dicha.

En realidad no se proporciona una forma única de descripción, sino un conjunto de métodos y herramientas para llevarla a cabo que tienen muy en cuenta iniciativas como TV Anytime, Dublin Core, Smpte Metadata Dictionary o EBU P/Meta, con las que trabajan codo con codo a través de los denominados "liaisons". De hecho, Mpeg-7 se puede combinar con otras iniciativas de descripción de recursos, como Dublin Core, sobre lo cual hay propuestas concretas ${ }^{7}$, o modificar los esquemas de descripción generados por $D D L$ para adaptarse a las necesidades concretas de alguno de estos estándares de metadatos.

Proporciona conjuntos de esquemas y descriptores estructurados para hacer frente a la descripción de diversos tipos de documentos y desde diferentes perspectivas. Básicamente divide en tres los tipos de información a tratar: sólo vídeo, sólo audio y multimedia. Aquí nos centraremos en éste último caso. Los esquemas de descripción multimedia (DMS) comprenden una serie de herramientas de descripción genéricas, que comparte con los esquemas de descripción de audio y los esquemas de descripción de vídeo, así como otra serie de aplicaciones específicamente multimedia sobre las que nos detendremos.

Existen algunos grupos de esquemas de descripción, como el de elementos base, que trabajan a bajo nivel y desde perspectivas técnicas, obteniendo una parte importante de los metadatos de forma automática. Es sobre estos elementos que se construyen el resto de los esquemas, pero no haremos referencia aquí a ellos por motivos de espacio. Sí hablaremos, aunque brevemente, de los de descripción y gestión del contenido, cuya mención parece más a propósito del tema que nos ocupa, reuniendo una gran parte de las herramientas que se encargarán de la descripción de documentos desde un punto de vista más documental.

Por una parte, tenemos las herramientas de gestión de los contenidos, que permiten introducir descriptores relacionados con los derechos de uso, precios, disponibilidad, historial de uso, y otra información financiero/administrativa, además de proporcionar información sobre el tipo de copia que se dispone (master o copias de distintos bitrates - bit rates o bit/seg- y calidades), sobre las condiciones de generación del contenido, autoría del documento y demás aspectos relacionados con la producción y generación de documentos audiovisuales.

\section{«Se llegó a pensar en rdf como el modelo para el desarrollo de DDL, algo en lo que tuvo mu- cho que ver, por otra parte, que $\mathrm{Xml}$ Schema estuviera todavía sólo parcialmente realizado»}

Por otra parte, el bloque de navegación y acceso contiene esquemas de descripción que permiten la elaboración de sumarios con acceso directo a los puntos del documento original al que se refieren, por lo que se proporciona una alta interacción con el usuario, que puede navegar a través de los documentos con la posibilidad de elaborar múltiples sumarios sin tener que duplicar los documentos asociados. Pueden ser tanto jerárquicos (con niveles progresivamente más específicos), como secuenciales, ordenados por criterios temporales. Otro de los conjuntos de herramientas es el de organización del contenido, que aporta esquemas de descripción para organizar y dar forma a colecciones de documentos, segmentos u objetos y describir sus propiedades comunes, lo que nos dota con capacidad para definir ontologías dentro de un archivo audiovisual, con la potencia que esto supone para su gestión. El grupo de interacción con el usuario, brinda, como su nombre indica, mecanismos para hacer posible la personalización de contenidos, así como para realizar estudios de usuarios.

Por último, convendría destacar el bloque que se ocupa de la descripción semántica y estructural de los contenidos audiovisuales. Por lo que hace a la estructural, se basa en la utilización de esquemas de descrip- 
ción de segmentos, que representan secciones de objetos audiovisuales, tanto temporales como espaciales, lo que permite definir divisiones del conjunto del documento por secuencias y a su vez dividirlas en escena, plano, fotograma u objeto, por ejemplo, o cualquier otra división, incluso relacionando segmentos de distintos documentos. También haría posible la descripción individualizada de cada una de las divisiones, combinando el esquema de descripción de segmentos con información referente al uso, creación, o anotaciones textuales, la interrelación de las mismas e incluso la utilización de herencia de propiedades entre divisiones y subdivisiones, todo lo cual proporciona una potente componente semántica al conjunto. La descripción conceptual de contenidos se hace a través de un esquema semántico, profundamente relacionado e interconectado con la descripción estructural de los contenidos, incluye objetos, conceptos, acontecimientos y las relaciones posibles entre dichos elementos.

\section{Rdf.}

Otro de los caminos que se ha seguido en la búsqueda de mecanismos de descripción adecuados ha sido adaptar rdf a las necesidades audiovisuales. Esto ocurrió durante las etapas iniciales de trabajo en el estándar Mpeg-7, durante las cuales se llegó a pensar en rdf como el modelo para el desarrollo de $D D L$, algo en lo que tuvo mucho que ver, por otra parte, que $\mathrm{Xml}$ Schema estuviera todavía sólo parcialmente realizado. Una vez desarrollado, se impuso a rdf y de hecho fue adoptado casi íntegramente por Mpeg-7 para la creación de su $D D L$.

Otras iniciativas tempranas son las que proponen conjuntos de metadatos como Dublin core, utilizando sintaxis $\mathrm{rdf}^{8}$. El problema fundamental de este acercamiento reside en la propia generalidad de Dublin core, por lo que existen adaptaciones al formato audiovisual a través de cualificadores o extensores de los campos originales de Dublin core ${ }^{9}$. Esto permite, en combina- ción con una sintaxis rdf, la descripción adaptada de documentos audiovisuales, que se puede aplicar a los distintos niveles de los que está compuesto un documento audiovisual (secuencias, escenas, planos, imágenes, objetos). Se propusieron algunos modelos ${ }^{10}$, pero la estabilización de Mpeg-7 parece haber hecho mella, y aunque algunos se han mantenido, otros han adoptado el nuevo estándar.

Un ejemplo del impacto de Mpeg-7 sobre la industria audiovisual podemos observarlo en Ibex, (Integrated broadband environment for personalized TV experience) un proyecto en el que se implicó la propia Sony Corporation y que comenzó utilizando rdf como lenguaje para la descripción de documentación audiovisual, intención que abandonó al consolidarse Mpeg- $7^{11}$.

Sin embargo, rdf todavía cuenta con algunas ventajas a su favor, como la sencillez de uso y su adaptabilidad al trabajo con metadatos, que pueden dar que hablar en el futuro. Actualmente las iniciativas para la descripción de recursos audiovisuales giran fundamentalmente en torno a Smil (Syncronized multimedia integration language) que supone el foco de interés principal del $W 3 C$ para el multimedia.

\section{Smil.}

Es un lenguaje para la creación de documentos multimedia, es decir, en formato xml que a su vez integran otros documentos a los que hacen referencia, como vídeos, secuencias de audio, texto en movimiento, etc. La especificación actual es la 2.0, que incorpora una forma bastante avanzada de describir metadatos. Con anterioridad, la versión 1.0 contaba sólo con un elemento, "meta", para descripciones bastante rudimentarias de contenidos multimedia. En la actualidad dispone de un módulo propio de metadatos para la descripción de su contenido, basado en la introducción de nuevos recursos de descripción utilizando rdf, cosa que permite la modularidad del lenguaje, y en general

\section{Versión online de EPI}

Existe una versión electrónica de El profesional de la información, de uso gratuito para la mayoría de los suscriptores (empresas, organismos, instituciones), que pueden acceder a través de internet a los textos completos y materiales gráficos publicados en la revista.

Más información en:

http://www.szp.swets.nl/szp/journals/pi- I I.htm

http://www.szp.swets.nl/szp/frameset.htm?url=/szp/eproducts/licence.htm http://www.extenza-eps.com/extenza/contentviewing/viewJournal.do?journalld=65 
la alta capacidad de interacción entre distintos lenguajes xml.

Las ventajas de combinar rdf con un lenguaje de presentación multimedia pueden encontrarse en la forma en la que éste crea documentos a partir de la conjunción de elementos dispersos, tratando de forma individual los distintos tipos de formatos para poder integrarlos en marcos más amplios desde los que se define su comportamiento y se describen sus contenidos. Un ejemplo sería la generación de compactados a partir de diferentes fragmentos de documentos, cosa que no se hace reuniendo en un documento nuevo todos estos fragmentos, sino llamándolos a través de Smil, que considera los aspectos temporales necesarios para poder hacer referencia a partes determinadas de documentos audiovisuales. Este documento Smil compuesto de "llamadas" a otros documentos, conlleva la descripción de cada uno de los fragmentos utilizando sintaxis rdf y un estándar de metadatos que sea soportado por rdf, lo que permite un alto grado de autonomía en la gestión de documentos.

Éste era un ejemplo de la utilidad de Smil, que ofrece algunas otras ventajas, como la facilidad de uso y que lo están convirtiendo en un formato de gestión de documentación multimedia atractivo.

\section{Ejemplo de elaboración de un archivo digital de documentación audiovisual}

En el marco del equipo de investigación Multidoc $^{12}$ estamos aplicando de forma experimental los estándares semánticos y sintácticos a los que nos hemos referido de forma sucinta a lo largo de este artículo. Nuestro objetivo es el de trabajar sobre un modelo unificado de tratamiento documental basado en sintaxis xml y en modelos semánticos surgidos de los esfuerzos de normalización de los distintos grupos de trabajo que en la actualidad están desarrollando modelos para la descripción de documentos.

Nuestro campo de experimentación es la documentación surgida a raíz de la puesta en marcha de la iniciativa Red Iberoamericana de Documentación Informativa. Se trata de un proyecto de carácter experimental en el que se pretenden investigar las diferentes vías de solución de un problema fundamental: la gestión de información multimedia en soporte electrónico. Evidentemente, el proyecto no pretende ser resolutivo sino prospectivo.

Existen unos requisitos mínimos, cumplidos los cuales el proyecto se consideraría un éxito. En primer lugar, la adquisición de un conocimiento de primera mano acerca del ámbito de la gestión de documentación multimedia, para lo que la investigación es una herramienta fundamental. En segundo lugar, se pretende diseñar e implementar un software a partir de las experiencias reunidas en la fase de investigación, y ponerlo al alcance de cualquier usuario o profesional a partir de su publicación bajo licencia $G N U$ en internet.

Desde luego, el punto de partida hace referencia a unas necesidades concretas que pasamos a detallar. Para empezar, la Red Iberoamericana de Documentación Informativa se extiende desde el punto de vista temático sobre áreas muy variadas, lo que impone ciertas exigencias en la elección de la semántica a utilizar en la descripción de sus contenidos. Existe una agitada polémica en el ámbito de los metadatos en torno a la dialéctica interoperabilidad/precisión; es decir, que en cada uno de los platillos de la balanza encontramos por una parte la necesidad de poder compartir nuestra información, y por otra la de describirla de la forma más adecuada a nuestros propósitos.

En el origen de muchas de las iniciativas de creación de conjuntos de metadatos está una o la otra, y en algunas ocasiones, ambas tendencias en liza. En el caso de los metadatos sobre documentación audiovisual la preferencia es generalmente a la precisión. Esto es así por los requisitos tanto técnicos como legales que deben cumplir los metadatos para las televisiones, pero también por los criterios de gestión de la documentación audiovisual, cuyos procesos se han ido refinando con el tiempo, exigiendo cada vez más definición en la descripción de los materiales.

En sentido contrario, existen iniciativas de tipo generalista, como Dublin core metadata initiative, que plantea un estándar ya reconocido de descripción de la documentación en el que la interoperabilidad, es decir, la capacidad de compartir, recuperar y operar sobre conjuntos de datos descritos bajo una misma plantilla es la prioridad.

\section{Notas}

1. Se puede obtener una idea más exacta de la magnitud del proyecto en la documentación proporcionada por la Biblioteca del Congreso, entre la que destaca el informe principal: Preserving our digital heritage: plan for the national digital information infrastructure and preservation program. http://www.digitalpreservation.gov/repor/ndiipp_plan.pdf

2. Patrimonio.es es una iniciativa auspiciada por el $\mathrm{M}^{\mathrm{o}}$ de Educación, Cultura y Deporte, el $\mathrm{M}^{\mathrm{o}}$ de Ciencia y Tecnología, la Unión Europea a través de los fondos de cohesión y la entidad pública estatal Red.es. Las diversas actuaciones previstas en el programa pueden ser consultadas en: http://www.patrimonio.es/primeras_actuaciones.html

3. Citado en: De Jong, Annemieke. Los metadatos en el entorno de la producción audiovisual. Una introducción. México D. F.: Netherlands Audiovisual Archive: 2001, p. 31.

4. Íbidem.

5. http://www.ebu.ch/departments/technical/pmc/pmc_meta.html

6. http://aaf.sourceforge.net/ 
7. Para una propuesta concreta en la que se combinan Dublin core y Mpeg-7, que aunque no se llevó a la práctica es representativa del modelo que queremos mostrar ver la nota $\mathrm{n}^{\mathrm{o}} 10$.

8. Se puede observar un estado del proyecto en:

http://dublincore.org/documents/2002/04/22/dcmes-xml

9. Para hacer lo propio con los metadatos cualificados o extendidos, podemos examinar:

http://dublincore.org/documents/dcq-rdf-xml/

10. Hunter, Jane; Armstrong, Liz. A comparison of schemas for video metadata representation.

http://www8.org/w8-papers/3c-hypermedia-video/comparison/

comparison.html

11. Gonno, Yoshihisa; Nishio, Fumihiko; Tsunoda, Tomohiro; Yamagishi, Yasuaki. Integrated broadband environment for personalized tv experience (Ibex)-Implementation study and practice.

http://www.acm.org/sigmm/mm2001/ep/gonno/

12. Servicio de documentación multimedia (Departamento de Biblioteconomía y Documentación UCM).

http://multidoc.rediris.es

\section{Bibliografía utilizada y webs con documentación consultada}

AAF Association.

http://www.aafassociation.org/

$B B C$ standard media exchange framework.

http://www.bbc.co.uk/guidelines/smef/

Data dictionary for audio/video metadata.

http://lcweb.loc.gov/rr/mopic/avprot/dbback2.html

De Jong, Annemieke. Los metadatos en el entorno de la producción audiovisual. Una introducción. México D. F.: Netherlands Audiovisual Archive, 2001.

Dublin core metadata initiative.

http://dublincore.org/

EBU Escort 2.4 system for the classification of RTV programmes.

http://www.ebu.ch/tech_escort2-4.pdf

EBU project group $P / F R A$.

http://www.ebu.ch/pmc fra.html

EBU P/Meta Metadata exchange scheme, V0.9

http://www.ebu.ch/pmc_meta.html

EBU technical review, 2000, September, n. 284.

http://www.ebu.ch/trev 284-hopper.pdf
Introduction to Mpeg-7 (v3.0).

http://ipsi.fhg.de/delite/Projects/MPEG7/Documents/W4325\%20M7\%20I ntro.htm

Material exchange format (MXF).

http://www.pro-mpeg.org

Metadata encoding and transmission standard (Mets).

http://www.loc.gov/standards/mets/

Metadata for long-term preservation.

http://www.kb.nl/coop/nedlib/results/D4.2/D4.2.htm

Mpeg-7 description definition language ( $D D L)$.

http://archive.dstc.edu.au/mpeg7-ddl/

Normes relatives aux métadonnées. En: Réseau canadien d'information sur le patrimoine. Canadá 2002.

http://www.chin.gc.ca/Francais/Normes/metadonnees_multimedia.html

Página oficial de $D D L$.

http://archive.dstc.edu.au/mpeg7-ddl/

Página oficial de Mpeg.

http://mpeg.telecomitalialab.com/

Overview of the Mpeg-7 standard (version 8.0).

http://ipsi.fhg.de/delite/Projects/MPEG7/Documents/N2467.html

Smpte metadata dictionary as specified in Smpte RP210a.

http://www.smpte-ra.org/mdd/index.html

TV-Anytime website.

http://www.tv-anytime.org

Vásquez, Cristian. Introducción a los metadatos.

http://www.dcc.uchile.cl/ cvasquez/meta_overview.html\#_Toc31706854

Video development initiative (ViDe) User's Guide: Dublin core application profile for digital video.

http://www.vide.net/workgroups/videoaccess/resources.shtml

Alfonso López Yepes; Rodrigo Sánchez Jiménez y José Ramón Pérez Agüera, Servicio de Documentación Multimedia (Multidoc), Departamento de Biblioteconomía y Documentación, Facultad de Ciencias de la Información, Universidad Complutense de Madrid.

alopez@ccinf.ucm.es

rodrigosj@mi.madritel.es

joseaguera@mundosmodernos.es

\section{Próximos temas especiales}

\section{Enero 2004 Minería de la web (web mining) Marzo 2004 Bibliotecas digitales Mayo $2004 \quad$ Usabilidad y arquitectura en la web}

Los interesados pueden remitir notas, artículos, propuestas, publicidad, comentarios, etc., sobre estos temas a:

epi@sarenet.es 